\section{Human factors}

\section{A wealth of accessible information}

Sir, I was pleased to see the launch of Human Factors and Patient Safety in Dentistry publicised in the $B D J(2020 ; 229: 11)$. Prior to my retirement in December 2019 I was Vice Chair of the Board which developed this resource. Written 'by dental team members for dental teams' it contains a wealth of accessible information. Teams may find it helpful to identify a lead member to promote use of the resource and to ensure the principles are embedded in the team's systems and processes. The resource can be accessed at www.humanfactors.dental and can additionally contribute to individual development and understanding.

L. Howells, Caerbryn, Wales https://doi.org/10.1038/s41415-020-2240-0

\section{Royal Colleges}

\section{Not entirely accurate}

Sir, I am writing in response to the recent interview with Dr Shelagh Farrell (BDJ 2020; 229: 164) which includes the claim there is no royal college for dentists. I too hold the MGDS qualification and was also part of the Advisory Committee for the inception of the FGDP, and indeed continue to support its evolution to a College. However, to claim there is currently no royal college for dentists is not entirely accurate.

In fact, all four Royal College of Surgeons (Edinburgh, England, Glasgow and Ireland) have dedicated Faculties of Dental Surgery. In particular, that of the Royal College of Surgeons of Edinburgh has been around for over 70 years and represents over 7,000 dentists from all over the world, ranging from general dental practitioners to specialist level. We are continually trying to broaden our reach and to encourage more dental professionals to join, including DCPs, through targeted advertising and stakeholder partnerships.

RCSEd's Faculty of Dental Surgery offers a range of quality assured diplomas, membership and fellowship qualifications, advocates on behalf of the industry and facilitates high levels of education for members to promote excellence in patient care.

In particular, the Faculty has played a crucial role in supporting its members over the last five months, by helping them understand the ever-changing regulations imposed as a result of COVID-19, and lobbying on their behalf on issues such as clearer guidance on and provision of PPE. As a Faculty, we are committed to working with the rest of the sector to ensure the best possible outcomes for dentists and patients, and I'd like to make it clear that everyone in dentistry is welcome to join.

P. Taylor, Dean Elect, Royal College of Surgeons of Edinburgh, Edinburgh, UK https://doi.org/10.1038/s41415-020-2241-z

\section{Oral health}

\section{Hazards of cocaine misuse}

Sir, you published a highly insightful article Cocaine and oral health in 2008 (BDJ 2008;

204: 365-369). We detail a case of this unusual presentation here with a brief literature review. A 48-year-old female presented with both nasal septal and hard palate perforation secondary to cocaine misuse (Fig. 1). Her history consisted of nasal congestion and obstruction which had deteriorated. She also developed facial pain, foul odour and sensation of food debris and fluids in her nasal cavity. Clinical examination demonstrated an obvious saddle nose deformity due to loss of nasal structural support. Intra-oral examination revealed partial anterior hard palate destruction leading to an oro-nasal fistula. On further questioning the patient admitted to a ten-year history of daily nasal cocaine use in addition to alcohol misuse.

Due to raised inflammatory markers and severe facial pain the patient was admitted to the ENT/maxillo-facial inpatient ward. She received a course of IV antibiotics and nasal douching. She was reviewed by the maxillofacial team and fitted with a cover plate to prevent passage through the oronasal fistula. The GP was asked to arrange substance misuse support for the patient to help with cocaine/alcohol cessation. The patient failed to engage with rehabilitation services and surgery was not advised due to the high risk of recurrence.

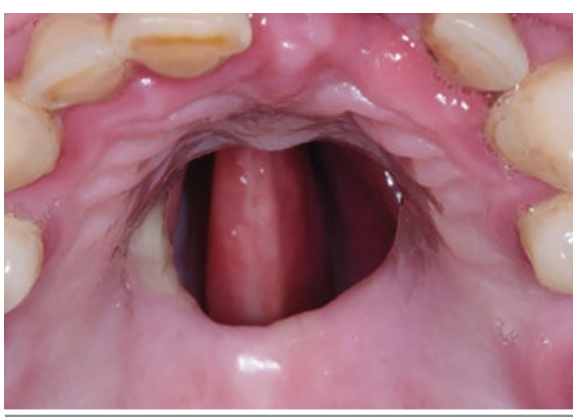

Fig. 1 Oro-nasal fistula showing a defect in the anterior hard palate extending to the nasal septum
Cocaine is one of the most commonly used narcotics and is a central nervous stimulant resulting in a short term increase in dopamine release. ${ }^{1,2}$ Well known for its uses of anaesthetic and pain relief its chemical structure has been altered to produce commonly used preparations such as lidocaine, one of the most frequently used topical and injectable local anaesthetics.

There are three common routes of cocaine administration: intravenous, insufflation and smoke inhalation. ${ }^{3}$ Prolonged misuse of cocaine can lead to a myriad of complications which can vary based on the route of administration. Nasal septal perforation is an uncommon presentation and is more commonly associated with trauma and previous septal surgery. ${ }^{4}$

Upon presentation of ulcerative lesions of the face, differential diagnoses must be considered including trauma, infection and neoplastic disease. Ulceration secondary to substance misuse is an uncommon occurrence but sadly the trend is on the rise. Orofacial lesions and destructive midline perforation is not exclusive to abusers of cocaine and physicians who are involved in the diagnosis and treatment of these lesions should be aware that a number of agents and pathophysiological causes can be associated with this appearance.

\section{H. Chaudhry, J. Stansfield, A. Camilleri,} Manchester, UK

\section{References}

1. Brownlow H A, Pappachan J. Pathophysiology of cocaine abuse. Eur J Anaesthesiol 2002; 19: 395.

2. Nestler E J. The neurobiology of cocaine addiction. $\mathrm{SC}$ Pract Perspect 2005; 3: 4-10

3. Jeffcoat A R, Perez-Reyes M, Hill J M, Sadler B M, Cook CE. Cocaine disposition in humans after intravenous injection, nasal insufflation (snorting), or smoking. Drug Metab Dispos 1989; 17: 153-159.

4. Metzinger S. Diagnosing and treating nasal septal perforations. Aesthet Surg J 2005; 25: 524-529. https://doi.org/10.1038/s41415-020-2242-y

\section{Eco-brushing}

Sir, as consumers are becoming environmentally conscious and the toll of greenhouse gases are apparent, people are understandably looking to reduce their carbon footprint. Currently the majority of toothpaste tubes are not recyclable with an estimated 260 million tubes contributing to landfill annually with biodegradation taking 500 years. ${ }^{1}$

While Colgate offers a nationwide recycling programme of toothpaste tubes, this is not commonly accepted by local councils. ${ }^{2}$ Therefore, for those keen to reduce waste, alternatives are sought but these often do not contain fluoride, which as we know, 
reduces cariogenic potential.

Toothpaste tablets, in glass jars, boxes or biodegradable bags, are available with certain lines containing 1,350 ppmF and above. This may be a happy medium to recommend to patients looking for plastic free alternatives. It may also be a more recognised form of toothpaste in the years to come.

\section{F. Chasma, Liverpool, G. Cousin, Blackburn}

\section{References}

1. Georganics. Zero Waste. Available at: https:// georganics.com/pages/eco-packaging (accessed 22 September 2020).

2. Colgate. The Colgate Oral Care Recycling Programme. Available at: https://www.terracycle.com/en-GB/ brigades/colgate-uk (accessed 3 July 2020).

https://doi.org/10.1038/s41415-020-2243-x

\section{Paediatric dentistry}

\section{SDF to the rescue}

Sir, we agree wholeheartedly with the letter from A. Remtulla, SDF - a game changer?, ${ }^{1}$ which describes the potential of silver diamine fluoride (SDF) within community dentistry and general dental practice as a treatment to arrest carious lesions and reduce the number of children requiring GA for extractions. The British Society of Paediatric Dentistry (BSPD) is campaigning for the technique to be licensed for the treatment of caries in addition to dentine hypersensitivity. We have created resources for colleagues to include a standard operating procedure, a consent form, a patient information leaflet, an explanatory PowerPoint and demonstration video, all of which can be found in the resources area of our website. ${ }^{2}$ We believe that the widespread use of SDF is part of the solution to improving children's oral health.

C. Stevens, Manchester, UK

\section{References}

1. Remtulla A. SDF - a game changer? Br Dent J 2020; 229: 4.

2. British Society of Paediatric Dentistry. Resources Available at: https://www.bspd.co.uk/Professionals/ Resources (accessed September 2020).

https://doi.org/10.1038/s41415-020-2244-9

\section{Endodontics}

\section{FDs' competence}

Sir, a survey was sent to foundation dentists (FDs) across South Yorkshire on endodontic working length determination (EWLD).

Despite only recently graduating, $41 \%$ of the 29 FDs in the survey had already changed their approach to WLD; $31 \%$ always obtained a working length radiograph, while $69 \%$ always obtained a master GP radiograph.

Despite electronic apex locators (EAL)

being considered by many to be an essential part of the modern endodontic armamentarium, providing greater accuracies, only 21 foundation dentists always used one. ${ }^{1}$ Factors limiting EAL performance include the type and amount of irrigant, ${ }^{2}$ file size ${ }^{3}$ and apical foramen size. ${ }^{4}$ Respondents indicated a lack of awareness regarding these factors: $34.5 \%$ were not aware of the influence of file size; $44.8 \%$ did not understand the influence of the type and amount of irrigant; and 55.2\% were not aware of how apical foramen size can affect EAL performance.

Mixed responses concerned how the FDs portrayed their experiences regarding teaching/training on how to use EALs. Thirteen (44.8\%) considered their education as average, ten (34.5\%) above average, four (13.8\%) below average and just two (6.9\%) as excellent. In support of the above results, a large majority (79.3\%) felt further teaching on EAL use would be very beneficial; $17.2 \%$ of limited benefit; $3.4 \%$ of no benefit.

It appears that FDs would embrace further education on the general topic of EWLD, with the majority (79.3\%) finding this proposition very beneficial and the remaining $20.7 \%$ suggesting additional teaching to be of limited benefit. My results highlight that FDs clearly appreciate the deficiencies in their knowledge and appear open to the idea of additional endodontic teaching. However, we must pinpoint why many $(58.6 \%)$ regarded their previous teaching/training as average or below average. In doing so we can prevent recurrence, guaranteeing both a better use of resources and maximising the learning potential for our young dental professionals.

W. Thorley, Sheffield, UK

\section{References}

1. Mandlik J, Shah N, Pawar K, Gupta P, Singh S, Shaik SA An in vivo evaluation of different methods of working length determination. J Contemp Dent Pract 2013; 14 644.

2. Tsesis I, Blazer T, Ben-Izhack $\mathrm{G}$ et al. The precision of electronic apex locators in working length determination: a systematic review and meta-analysis of the literature. J Endod 2015; 41: 1818-1823.

3. Orosco F A, da Silva G F, Weckwerth P H et al. Influence of different sized files on the accuracy of two electronic apex locators. Aust Endod J 2018; 44: 251-254.
4. Kolanu SK, Bolla N, Varri S, Thummu J, Vemuri S, Mandava P. Evaluation of correlation between apical diameter and file size using Propex Pixi apex locator. J Clin Diag Res 2014; 8: 18-20.

https://doi.org/10.1038/s41415-020-2245-8

\section{Mouth cancer}

\section{Online mouth cancer module}

Sir, the paper by Araghi et al. (BDJ 2020; 228: 693) reminds us all that we need to remain vigilant for any changes that should raise our index of suspicion for cancer of the mouth. Greater awareness through use of the various toolkits designed to educate doctors and dentists about 'the correct lesions' to be sent in on the two-week referral system should also include mention of the $B M J$ online learning module for oral cancer. This was originally sponsored by the Ben Walton Trust (BWT), and is available online. ${ }^{1}$ Readers may be interested to know that the BWT funds were transferred to the Royal College of Physicians \& Surgeons of Glasgow in 2017 where this resource is still available to support the original ethos of the Trust (to raise both public and professional awareness of mouth cancer).

The results of Araghi et al.'s audit would seem to suggest the need for this is as great as ever.

G. Ogden, Dundee, UK

\section{Reference}

1. BMJ Learning. Mouth cancer: recognising it and referring early. 19 November 2015. Available at: https://learning.bmj.com/learning/moduleintro/mouth-cancer-recognising-referring-early. $\mathrm{html}$ ? locale=en_GB\&moduleld=10015809 (accessed 22 September 2020).

https://doi.org/10.1038/s41415-020-2246-7

\section{Correction to: Nursing oral care}

The original article can be found online at https://doi.org/10.1038/ s41415-020-1901-3

Author's correction note:

Letter Br Dent J 2020; 229: 3.

When this article was published $\mathrm{R}$. Suffern was omitted from the author list. The author list should have read:

R. Smith, A. Panchal, R. Suffern, I. Politi, M. Barker, T. Pepper, and F. Ryba. This has been corrected online.

The authors apologise for any inconvenience caused.

https://doi.org/10.1038/s41415-020-2230-2 